\title{
Altered emotional recognition and expression in patients with Parkinson's disease
}

\author{
This article was published in the following Dove Press journal: \\ Neuropsychiatric Disease and Treatment \\ Number of times this article has been viewed
}

\author{
Yazhou Jin* \\ Zhiqi Mao* \\ Zhipei Ling \\ Xin Xu \\ Zhiyuan Zhang \\ Xinguang Yu \\ Department of Neurosurgery, \\ People's Liberation Army General \\ Hospital, Beijing, People's Republic \\ of China \\ *These authors contributed equally \\ to this work
}

\begin{abstract}
Background: Parkinson's disease (PD) patients exhibit deficits in emotional recognition and expression abilities, including emotional faces and voices. The aim of this study was to explore emotional processing in pre-deep brain stimulation (pre-DBS) PD patients using two sensory modalities (visual and auditory).
\end{abstract}

Methods: Fifteen PD patients who needed DBS surgery and 15 healthy, age- and gendermatched controls were recruited as participants. All participants were assessed by the Karolinska Directed Emotional Faces database 50 Faces Recognition test. Vocal recognition was evaluated by the Montreal Affective Voices database 50 Voices Recognition test. For emotional facial expression, the participants were asked to imitate five basic emotions (neutral, happiness, anger, fear, and sadness). The subjects were required to express nonverbal vocalizations of the five basic emotions. Fifteen Chinese native speakers were recruited as decoders. We recorded the accuracy of the responses, reaction time, and confidence level.

Results: For emotional recognition and expression, the PD group scored lower on both facial and vocal emotional processing than did the healthy control group. There were significant differences between the two groups in both reaction time and confidence level. A significant relationship was also found between emotional recognition and emotional expression when considering all participants between the two groups together.

Conclusion: The PD group exhibited poorer performance on both the recognition and expression tasks. Facial emotion deficits and vocal emotion abnormalities were associated with each other. In addition, our data allow us to speculate that emotional recognition and expression may share a common system.

Keywords: Parkinson's disease, emotional recognition, emotional expression, visual, auditory

\section{Introduction}

Traditionally, PD is characterized as a movement disorder and neurodegenerative disease. However, PD patients may exhibit marked cognitive decline and emotional impairment in the moderate and advanced stages of the disease. ${ }^{1}$ Characteristic pathological changes in PD patients include degeneration of the substantia nigra and loss of dopamine. These changes affect the neural circuits from the basal ganglia to the thalamus and prefrontal cortex, which are associated with movement, and cognitive and emotional functions. ${ }^{2-5}$ In addition, other important brain structures, such as the amygdala and cingulate cortex, are involved. ${ }^{6}$

Accurately understanding the emotional intention of others is very important in interpersonal communication. Emotional recognition dysfunction impairs interpersonal relationships and lowers quality of life. As PD progresses, patients are more dependent on their caregivers. Complete emotional cognitive ability is necessary to maintain close relationships. Emotional cognition includes the recognition of emotional faces
Department of Neurosurgery, People's Liberation Army General Hospital, Fuxing Road 28, Haidian District, Beijing 100853 , People's Republic of China

Tel +8610638939

Email xinguangyu30I@I63.com 
and voices. PD patients show varying degrees of decline in these two aspects, and this decline is closely associated with age, gender, disease duration, and drug use. ${ }^{7-9}$ However, the decline of emotional recognition ability is unrelated to dementia or depression status.

In addition, emotional expression ability, including expression of emotional faces and voices, is decreased in PD patients. ${ }^{10-12}$ A proportion of PD patients cannot exhibit emotions or exert normal control over their throat muscles. Rusz et al suggested that the reduced ability to vocally express emotions can be found even in the early stage of PD. ${ }^{13}$ Electromyography studies have recorded delayed onset and reduced amplitude of facial muscles when patients spontaneously mimic the facial expressions of others. ${ }^{14}$ The inability to express emotional intentions and feelings in verbal or nonverbal communication is called emotional dysprosody. ${ }^{15}$ Low volume, flat and nonemotional pitch, and abnormal speech rate are characteristics of the speech of PD patients.

PD therapies include anti-PD medicine and surgical intervention. However, drug therapies are only effective for the early stage of PD. For moderate- and advanced-stage PD patients, DBS is an effective treatment that can considerably improve motor symptoms. However, surgeons have different opinions regarding target selection in DBS. The most commonly used targets in the clinic include the STN and globus pallidus interna. ${ }^{16}$ Although this supposition has not been confirmed, STNDBS may lead to acquired deficits in emotional processing. ${ }^{17,18}$ To understand the baseline emotional state of DBS candidates, we recruited a group of pre-DBS patients in this study. ${ }^{12}$

Since both emotional recognition and expression are impaired in PD patients, we hypothesized that these two aspects may share a common nervous system. In addition, another important question is whether facial emotion deficits and vocal emotion abnormalities are associated in pre-DBS PD.

We chose five basic human emotions: happiness, anger, sadness, fear, and neutral. Based on previous studies, two other basic human emotions, disgust and surprise, were eliminated because the recognition rate of these two emotions is relatively low. ${ }^{19,20}$ The aim of this study was to explore emotional processing in advanced PD patients who were candidates for DBS using two sensory modalities (visual and auditory). These results will provide insights into the mechanisms of emotional processing alteration and DBS in PD.

\section{Methods}

\section{Participants}

A total of 15 PD patients who needed DBS surgery in the People's Liberation Army General Hospital were recruited as participants. The average age was 61.73 years $(\mathrm{SD}=8.59)$, ranging from 50 to 75 years. The number of average education years was $8.8(\mathrm{SD}=3.30)$. The inclusion criteria were as follows: a diagnosis of advanced PD; poor symptom control using dopaminergic replacement therapy; issues affecting normal work or life; absence of cognitive dysfunction or dementia (score $>26$ on the Mini Mental State Examination and $>24$ on the Montreal Cognitive Assessment); and absence of depression (diagnosed according to the Hamilton Depression Scale), anxiety (diagnosed according to the Hamilton Anxiety Scale), or other psychiatric or brain organic disease. Fifteen healthy, age- and gender-matched controls were recruited as the control group. The average age was 61.67 years $(S D=7.75)$, ranging from 45 to 70 years. The number of average education years was $8.6(\mathrm{SD}=3.25)$. We also tested the control group using the cognitive and mental scales to exclude cognitive dysfunction or other psychiatric states. All participants' data were collected carefully. For patients, clinical data, such as disease duration, levodopa equivalent daily dose, Unified Parkinson's Disease Rating Scale score, or Hoehn and Yahr staging scale, were also included. Group differences in demographic, neuropsychological, and clinical characteristics were analyzed with an independent two-sample Student's $t$-test. ${ }^{21}$ The results are shown in Table 1.

\section{Procedure}

The experimental procedure contained three main experiments. The first experiment included emotional facial recognition and facial expression. The second experiment focused on emotional vocal recognition and vocal expression. The third experiment was concerned with emotional facial and vocal expression perception. The whole experiment

Table I Demographic and clinical data of participant groups

\begin{tabular}{llll}
\hline & PD $(\mathbf{n}=\mathbf{I 5})$ & HC $(\mathbf{n}=\mathbf{I 5})$ & P-values \\
\hline Gender ratio & $4 \mathrm{~F} / \mathrm{IIM}$ & $4 \mathrm{~F} / \mathrm{IIM}$ & \\
Age (years) & $61.73 \pm 8.59$ & $6 I .67 \pm 7.75$ & 0.98 \\
Education (years) & $8.8 \pm 3.30$ & $8.6 \pm 3.25$ & 0.87 \\
MMSE & $28.07 \pm 0.88$ & $28.13 \pm 0.92$ & 0.84 \\
MOCA & $26.2 \pm 1.08$ & $26.93 \pm 0.96$ & 0.06 \\
Disease duration (years) & $7.93 \pm 2.31$ & & \\
H\&Y & $2.53 \pm 0.74$ & & \\
LEDD & $393.96 \pm 199.69$ & & \\
UPDRS III & $29.87 \pm 15.63$ & & \\
\hline
\end{tabular}

Notes: Data presented as mean \pm SD. $P$-values $<0.05$ were considered statistically significant.

Abbreviations: PD, Parkinson's disease; HC, healthy control; MMSE, Mini Mental State Examination; MOCA, Montreal Cognitive Assessment; H\&Y, Hoehn and Yahr staging scale; LEDD, levodopa equivalent daily dose; UPDRS, Unified Parkinson's Disease Rating Scale. 
was conducted in a quiet, softly lit room. All patients took anti-PD drugs before the experimental procedure ("on" medication state).

\section{Experiment I: emotional facial and vocal recognition}

All participants (PD and HC) were assessed by the KDEF database 50 Faces Recognition test. ${ }^{20}$ Face pictures of 10 actors expressing five basic emotions (neutral, happiness, anger, fear, and sadness) were randomly presented on a computer screen, one at a time. The participants were asked to distinguish the correct emotion from a five forced-choice response format. RT was unlimited due to the movement disorder symptoms of the PD patients, but we encouraged the participants to make their choices quickly. The test provides scores out of 10 for recognizing each basic emotion and yields a score out of a maximum of 50 for recognizing all five emotions. To better understand the recognition test, participants were asked to describe each emotional type before the test.

Vocal recognition was evaluated by the MAV database 50 Voices Recognition test. ${ }^{22}$ Similar to the facial recognition test, five basic emotions (neutral, happiness, anger, fear, and sadness) were included. These nonverbal vocalizations of 10 actors were randomly played, one at a time. The participants were then asked to listen to each recording carefully while wearing headphones at a comfortable volume level and make a choice in a five forced-choice response format. The participants were also asked to describe each emotional type.

All trials were run with E-prime 2.0 software. Each trial sequence consisted of 1) the presentation of a fixation cross on the center of computer screen for 1,000 ms, 2) display of a picture or voice, and 3) a five forced-choice response format, indicating that an emotion judgment decision should be made.

\section{Experiment 2: emotional facial and vocal expression}

For the emotional facial expression task, the participants were asked to imitate five basic emotions (neutral, happiness, anger, fear, and sadness). A video recorder was fixed in front of the participants, and the frame included their faces, hair, and shoulders. Each emotional imitation process was recorded for at least 4 seconds. Four pictures were captured for each emotion and then numbered. One picture best expressing an emotion was chosen by two authors. Another author was included if the two authors held different opinions.
However, the first picture was selected if all four pictures did not express emotions. To ensure that the participants understood the task, the first author asked the participants to describe an example for each emotion.

Similarly, the subjects were required to express nonverbal voices of five basic emotions. The recording processes occurred under the same condition, and the recording parameters were consistent (sampling rate at $48,000 \mathrm{~Hz}$, mono channel, resolution ratio at 16 bits). The materials were recorded using Adobe Audition 3.0 software as the operating platform and were edited into sound files in WAV format. The duration of the sound files of each emotional type was 1 second. To meet the demands of noise reduction, a 6-second blank section was recorded before each recording process. To ensure that the participants understood the task and had adequate expressive abilities, we described an example of a scenario for each situation ("What do you say when you feel happiness, sadness, fear, anger, and neutral?"). The structure of the nonverbal voices here was similar to the MAV database used in experiment 1 .

\section{Experiment 3: perception of emotional facial and vocal expression}

Fifteen Chinese native speakers were recruited as decoders (eight males, seven females). The average age was 26.88 years $(\mathrm{SD}=2.09)$, and their ages ranged from 25 to 30 years. They were all graduate students in the People's Liberation Army General Hospital and had been living in the People's Republic of China since birth. The average number of years of education was 20.27 ( $\mathrm{SD}=0.88$ ). Their native language was Chinese, and their second language was English. They had normal hearing without any nervous system disease and related medication history. All participants volunteered free of charge. These decoders evaluated the expression of the two groups (PD and HC) while sitting in front of a computer screen at a distance of $60 \mathrm{~cm}$. The 15 decoders were blinded to the diagnosis.

The pictures were divided into two groups (PD and $\mathrm{HC}$ ). Then, each picture was randomly presented on a computer screen, one at a time. The decoders were required to identify the emotional type in a five forced-choice response format (neutral, happiness, anger, fear, and sadness). The decoders assigned one point for a correct response and zero point for an incorrect response. The RT and accuracy rate of each decoder were calculated. The decoders were also asked to rate their CL for each choice (five points, very high; four points, high; three points, mild; two points, little; one point, very little). Furthermore, the authors offered an alternative option "zero point" if decoders had no confidence in their choices. 
For the perception test of emotional vocal expression, the experimental procedures were analogous. Three parameters were calculated, including RT, accuracy rate, and CL. Volume was adjusted to a comfortable level before the vocal perception test.

When analyzing the test results, we needed to examine the reliability of the accuracy rate. Additionally, interrater reliability was assessed by calculating Cronbach's alpha.

All perception trials were run with E-prime 2.0 software. After the perception test of emotional facial expression, the decoders had 5 minutes of rest before the vocal perception test.

\section{Ethical considerations}

The ethics committee of the People's Liberation Army General Hospital approved the research protocol. All participants agreed to participate in the study and signed the informed consent form.

\section{Statistical analysis}

KDEF sub-scores were analyzed by ANOVA with a $2 \times 5$ factorial design (between-subjects factor: $\mathrm{PD}$ and $\mathrm{HC}$; within-subjects factor: neutral, happiness, anger, fear, and sadness). Two separate $2 \times 5$ ANOVAs were performed for the variables CL and RT, with "group" (PD and $\mathrm{HC}$ ) as a between-subjects factor and "emotion" (neutral, happiness, anger, fear, and sadness) as a within-subjects factor. The linear relation between CL and RT was also analyzed. We designed analogous statistical analyses to evaluate the test of vocal recognition and the perception of facial and vocal expression. To determine the differences between groups, post hoc unpaired $t$-tests were performed. Before variance analysis, tests of normality and homogeneity of variance were applied. Mann-Whitney $U$ tests were used if the normality and homogeneity of variance assumptions were not satisfied. $P$-values $\leq 0.05$ were considered statistically significant.

The relationship between facial emotional recognition (KDEF total scores and sub-scores) and facial emotional expression (facial expression total scores and sub-scores) was assessed by Pearson correlation tests. Similarly, Pearson correlation tests were used to evaluate the relationship between vocal emotional recognition and vocal emotional expression. In addition, to determine whether a relationship exists between two sensory modalities (visual and auditory), we separately analyzed the correlation between facial emotional tasks (recognition and expression) and vocal emotional tasks (recognition and expression) using Pearson correlation tests. However, the data were analyzed by Spearman correlation tests instead of Pearson correlation tests if normality assumptions were not satisfied. All statistical analyses were performed using the SPSS 17.0 software package.

\section{Results}

\section{Emotional recognition in PD and $\mathrm{HC}$}

For facial emotional recognition, the ANOVA revealed significant main effects of the factors "group" $(F[1,99]=12.733$, $P=0.001$; Figure 1A) and "emotion" $(F[4,99]=24.637$, $P<0.001$; Figure 1B), with no significant interaction between the two factors. For vocal emotional recognition, significant main effects of the factors "group" $(F[1,99]=6.673, P=0.011$; Figure $1 C)$ and "emotion" $(F[4,99]=32.102, P<0.001$; Figure 1D) emerged with the ANOVA, with no significant interaction between the two factors. Post hoc $t$-tests revealed significantly lower scores for the KDEF sub-score of sadness in the PD group than those in the HC group $(P<0.001)$. The MAV sub-score of neutral in the PD group was lower than that in the HC group $(P<0.001)$. There were no other significant differences between the two groups (all $P>0.05$ ). The results are shown in Table 2.

\section{Emotional expression in PD and HC}

The Cronbach's alpha values in the perception test were all higher than 0.90 , indicating the accuracy rate results had high interrater reliability. For facial expression, we found main effects of "group" $(F[1,149]=9.022, P=0.003$; Figure 2A) and "emotion" $(F[4,149]=12.291, P<0.001$; Figure $2 \mathrm{C}$ ). These results were significantly different. For vocal expression, there were significant main effects of "group" $(F[1,149]=5.348, P=0.022$; Figure $2 \mathrm{~B})$ and "emotion" $(F[4,149]=10,467, P<0.001$; Figure $2 \mathrm{D})$, and these results were significantly different. Post hoc $t$-tests revealed that vocal expressions of neutral and anger were less recognized by the PD group than by the HC group $(P<0.05)$. There were no other significant differences between the two groups $(P>0.05)$. The results are shown in Table 3.

\section{RT and $C L$ in the emotional expression test}

As the normality and homogeneity of variance assumptions of RT and CL were not satisfied, Mann-Whitney $U$ tests were used for the analysis. The overall RT of the PD group was significantly longer than that of the HC group in the vocal emotion expression test $(P<0.05)$ (Figure 3C), while the overall RT was similar between the two groups in the facial emotion expression test $(P>0.05)$ (Figure 3A). Post hoc Mann-Whitney $U$ tests revealed a significantly longer RT for 
A

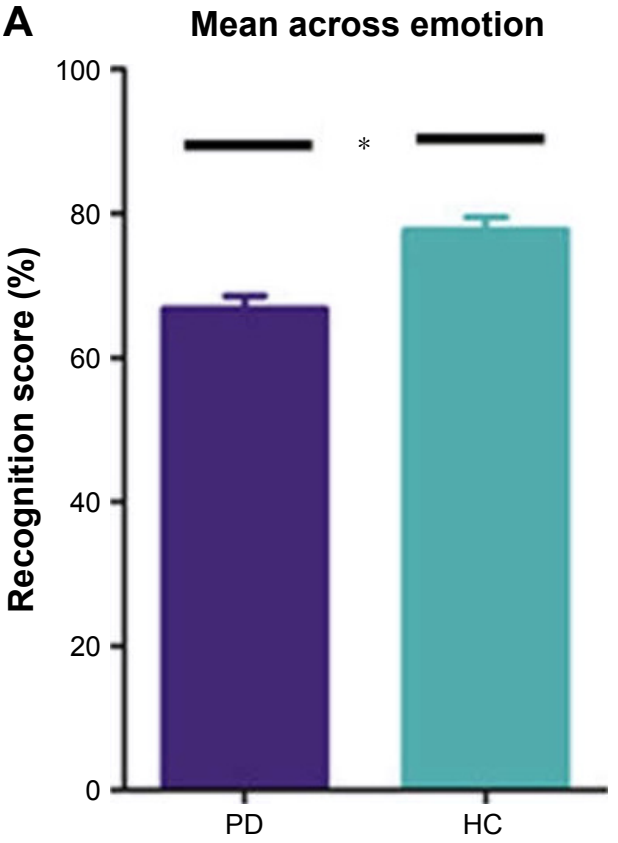

C

Mean across emotion

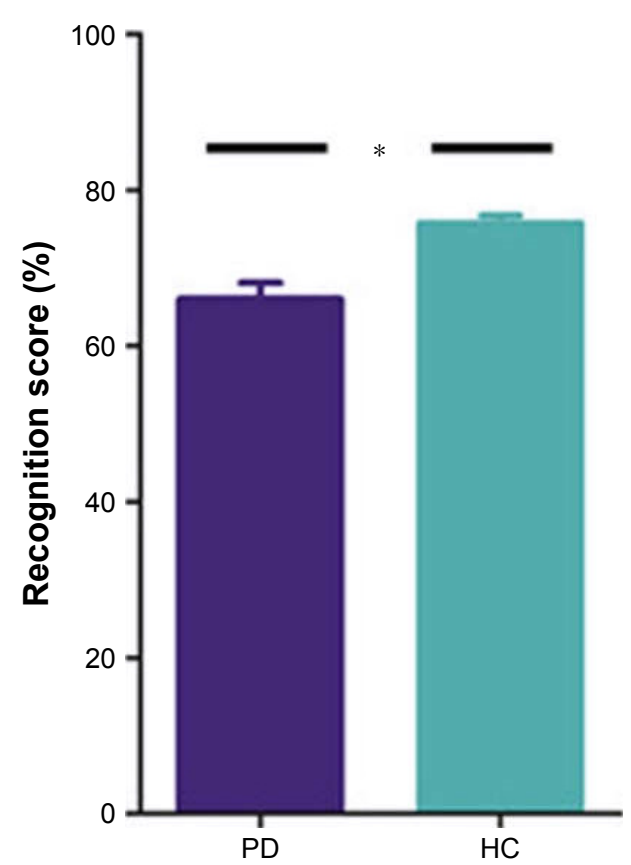

B

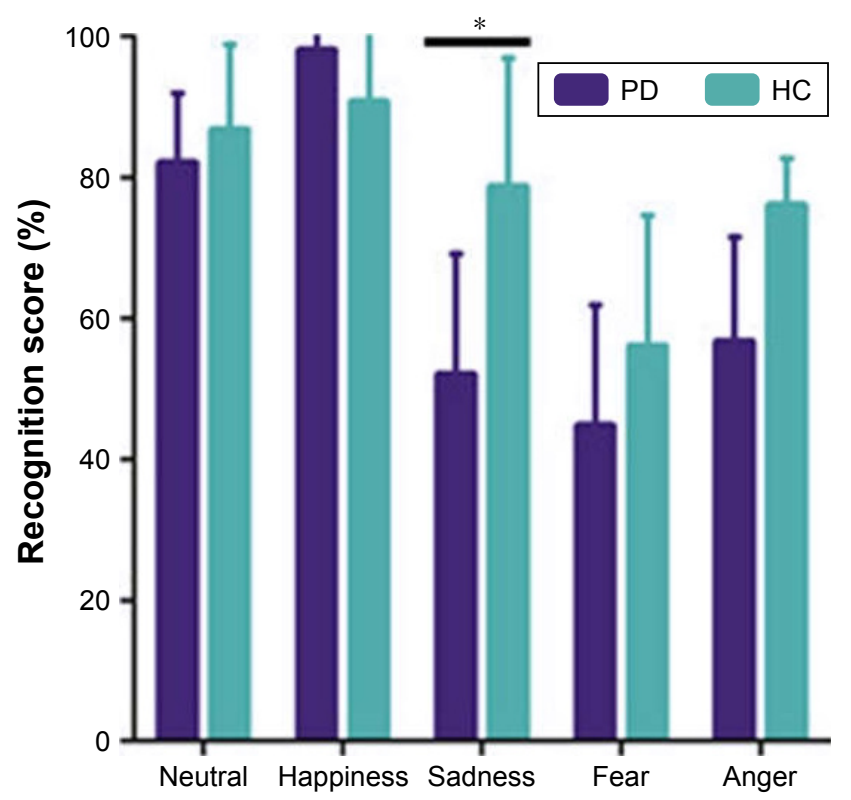

D

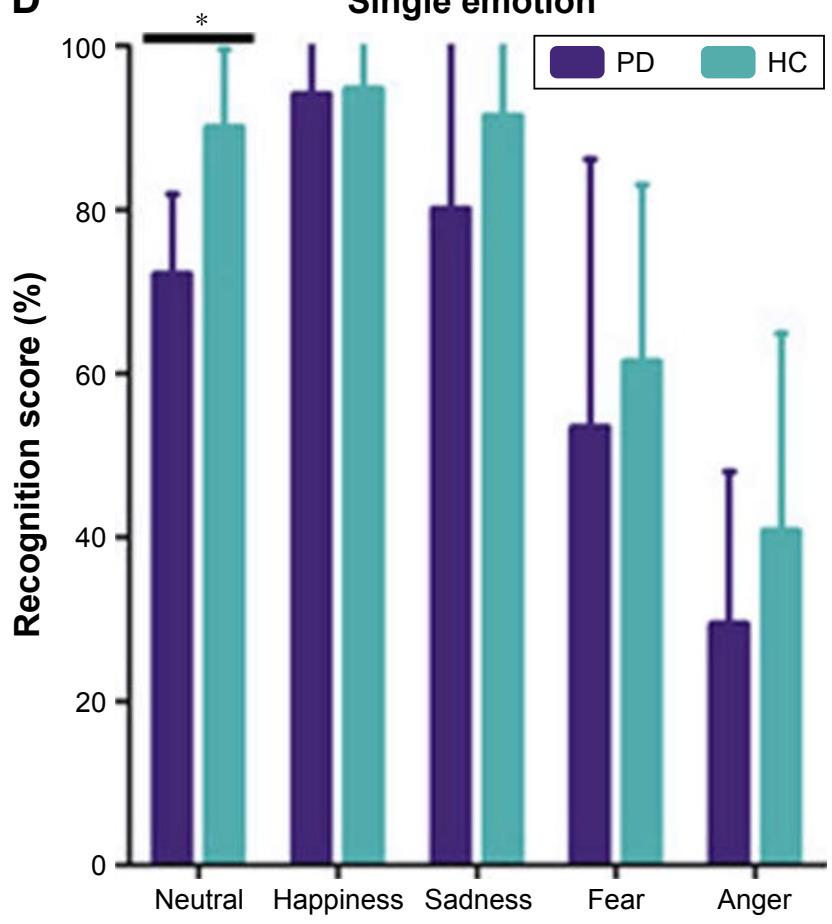

Figure I Facial emotion recognition task for the PD group (dark blue) and the HC group (light blue): (A) total score for all emotions and (B) sub-scores for single emotion. Vocal emotion recognition task for the PD group (dark blue) and the HC group (light blue): (C) total score for all emotions and (D) sub-scores for single emotion. Higher scores represent better performance. Data presented as mean \pm SD. Asterisks indicate a statistical difference between groups $(P<0.05)$.

Abbreviations: PD, Parkinson's disease; HC, healthy control.

the facial emotion expression test of happiness and fear in the PD group than that in the HC group $(P<0.05)$ (Figure 3B) and for the vocal emotion expression test of neutral and happiness in the PD group $(P<0.05)$ (Figure 3D). The RT for other emotions did not differ between the two groups. The results are shown in Table 4.
The raters were more confident in their choices when evaluating $\mathrm{HC}$ pictures and voices than when evaluating PD pictures and voices $(P<0.05)$ (Figure 4$)$. The results are shown in Table 5.

Spearman correlation analysis showed a weak correlation between RT and CL not only for facial emotional expression 
Table 2 Emotional recognition score in PD and HC

\begin{tabular}{llll}
\hline Recognition score $(\%)$ & PD $(\mathbf{n}=\mathbf{I 5})$ & HC $(\mathbf{n}=\mathbf{I 5})$ & P-values \\
\hline KDEF total score & $55.56 \pm 3.73$ & $72.00 \pm 3.70$ & $0.000^{*}$ \\
Neutral $(n=10)$ & $72.00 \pm 9.84$ & $90 \pm 9.56$ & 0.361 \\
Happiness $(n=10)$ & $94.00 \pm 11.09$ & $94.67 \pm 12.49$ & 0.127 \\
Sadness $(n=10)$ & $80.00 \pm 25.72$ & $91.33 \pm 10.45$ & $0.003^{*}$ \\
Fear $(n=10)$ & $53.33 \pm 32.81$ & $61.33 \pm 21.73$ & 0.175 \\
Anger $(n=10)$ & $29.33 \pm 18.65$ & $40.67 \pm 24.23$ & $0.016^{*}$ \\
MAV total score & $65.87 \pm 8.53$ & $75.60 \pm 4.29$ & $0.000^{*}$ \\
Neutral $(n=10)$ & $82.00 \pm 9.96$ & $86.67 \pm 12.17$ & $0.00 I^{*}$ \\
Happiness $(n=10)$ & $98.00 \pm 4.50$ & $90.67 \pm 13.77$ & 0.901 \\
Sadness $(n=10)$ & $52.00 \pm 17.16$ & $78.67 \pm 18.27$ & 0.213 \\
Fear $(n=10)$ & $44.67 \pm 17.23$ & $56.00 \pm 18.65$ & 0.528 \\
Anger $(n=10)$ & $56.67 \pm 14.82$ & $76.00 \pm 6.67$ & 0.256 \\
\hline
\end{tabular}

Notes: Data presented as mean \pm SD. Asterisks indicate a statistical difference between groups $(P<0.05)$.

Abbreviations: PD, Parkinson's disease; HC, healthy control; KDEF, Karolinska Directed Emotional Faces; MAV, Montreal Affective Voices. in the $\mathrm{HC}$ group but also for vocal emotional expression in the PD and HC groups (all $P<0.01$ ) (Figure 5A, C, and D). However, no relationship was found for facial emotional expression in the PD group $(P>0.05)$ (Figure 5B). The results are shown in Table 6.

\section{Relationship between emotional recognition and emotional expression}

When considering all participants between the two groups together, the Pearson's correlation analysis revealed a significant relationship (facial recognition task and vocal recognition task: $r=0.436, P=0.016$ [Figure 6A]; facial recognition task and facial expression task: $r=0.436, P=0.016$ [Figure 6B]; vocal recognition task and vocal expression
A

Mean across emotion

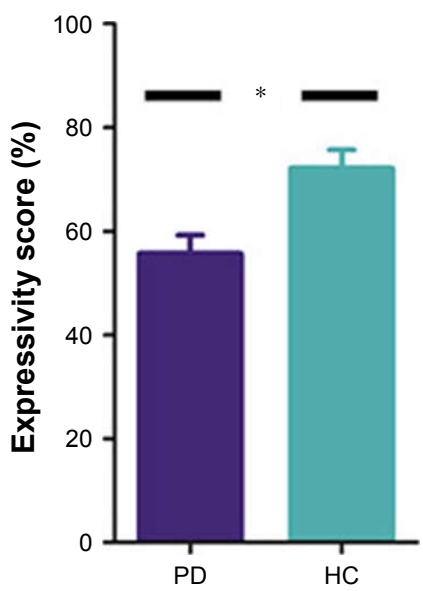

C

Mean across emotion

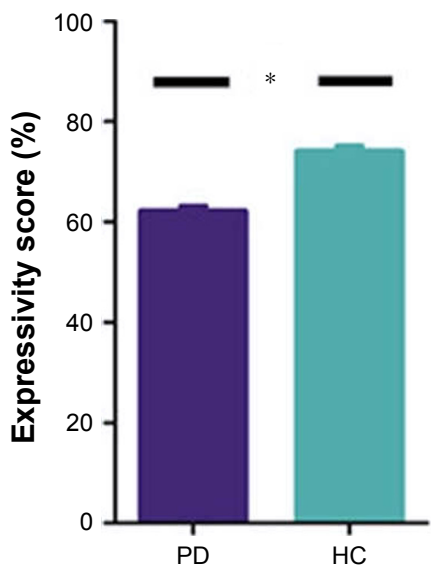

B Single emotion
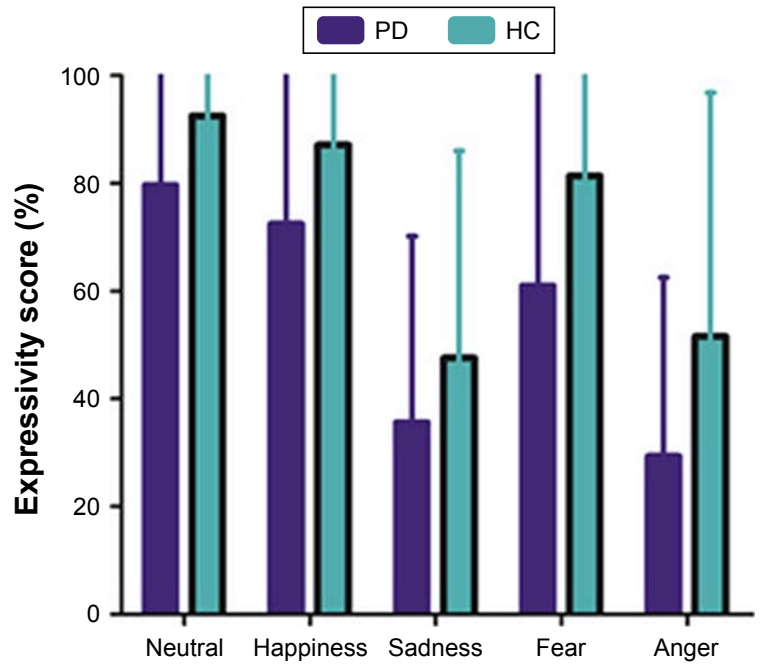

D Single emotion

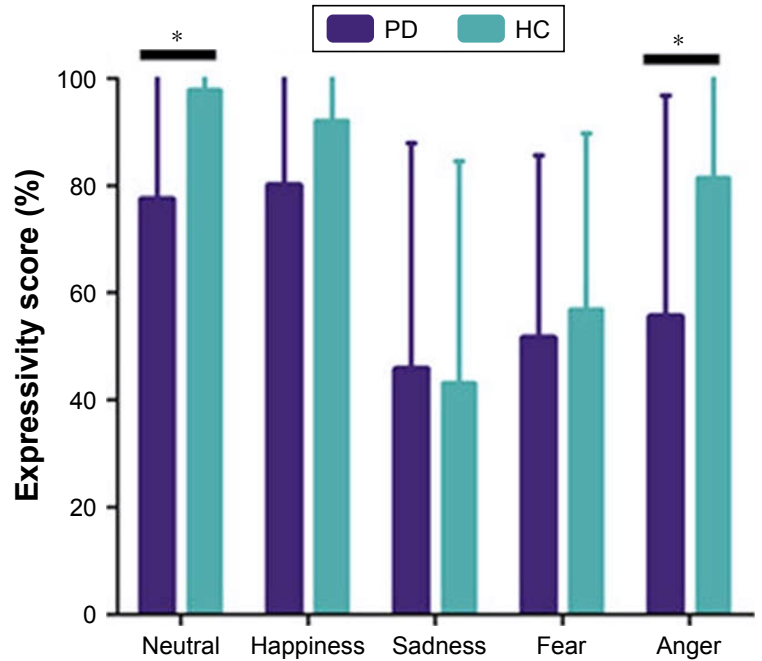

Figure 2 Facial emotion expression task for the PD group (dark blue) and the HC group (light blue): (A) total score for all emotions and (B) sub-scores for single emotion. Vocal emotion expression task for the PD group (dark blue) and the HC group (light blue): (C) total score for all emotions and (D) sub-scores for single emotion. Higher scores represent better performance. Data presented as mean \pm SD. Asterisks indicate a statistical difference between groups $(P<0.05)$.

Abbreviations: PD, Parkinson's disease; HC, healthy control. 
Table 3 Emotional expressivity score in PD and HC

\begin{tabular}{llll}
\hline Expressivity score (\%) & PD $(\mathbf{n}=\mathbf{1 5})$ & HC $(\mathbf{n}=\mathbf{I 5})$ & $P$-values \\
\hline Facial total score & $55.56 \pm 3.73$ & $72.00 \pm 3.70$ & $0.000^{*}$ \\
Neutral $(n=15)$ & $79.56 \pm 27.43$ & $92.44 \pm 10.04$ & 0.098 \\
Happiness $(n=15)$ & $72.44 \pm 42.00$ & $87.11 \pm 26.12$ & 0.261 \\
Sadness $(n=15)$ & $35.56 \pm 34.61$ & $47.56 \pm 38.45$ & 0.377 \\
Fear $(n=15)$ & $60.89 \pm 43.27$ & $81.33 \pm 22.85$ & 0.117 \\
Anger $(n=15)$ & $29.33 \pm 33.12$ & $51.16 \pm 45.25$ & 0.136 \\
Vocal total score & $62.04 \pm 1.18$ & $73.88 \pm 4.65$ & $0.000^{*}$ \\
Neutral $(n=15)$ & $77.38 \pm 32.15$ & $97.80 \pm 6.98$ & $0.023^{*}$ \\
Happiness $(n=15)$ & $80.00 \pm 34.09$ & $92.00 \pm 11.60$ & 0.207 \\
Sadness $(n=15)$ & $45.78 \pm 42.08$ & $43.11 \pm 41.39$ & 0.862 \\
Fear $(n=15)$ & $51.56 \pm 34.04$ & $56.89 \pm 32.84$ & 0.666 \\
Anger $(n=15)$ & $55.56 \pm 41.15$ & $81.33 \pm 24.97$ & $0.047^{*}$ \\
\hline
\end{tabular}

Notes: Data presented as mean \pm SD. Asterisks indicate a statistical difference between groups $(P<0.05)$.

Abbreviations: $\mathrm{PD}$, Parkinson's disease; $\mathrm{HC}$, healthy control. task: $r=0.891, P<0.01$ [Figure 6C]; facial expression task and vocal expression task: $r=0.391, P=0.033$ [Figure 6D]). The results are shown in Table 7.

\section{Discussion}

Compared with HCs, PD patients exhibited impaired emotional recognition and expression. Although not confirmed, alterations in brain areas are responsible for altered emotional processing, including recognition and expression. Brain areas such as the amygdala, prefrontal cortex, and the basal ganglia are involved. Studies have found that the amygdala and orbitofrontal cortex are associated with fear conditioning. ${ }^{23-25}$ In addition, diminished motor function may further contribute to impaired emotional expression processing. Delayed onset
A

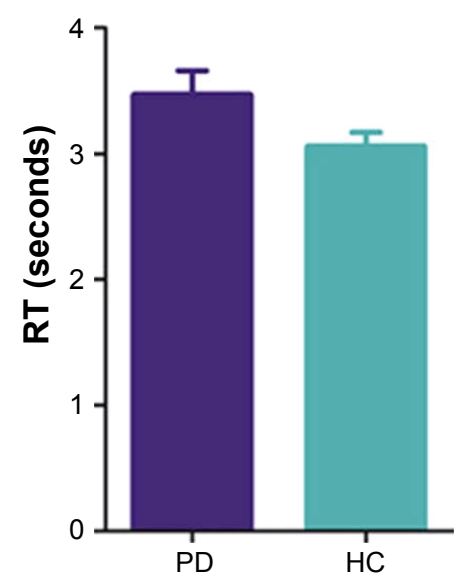

C

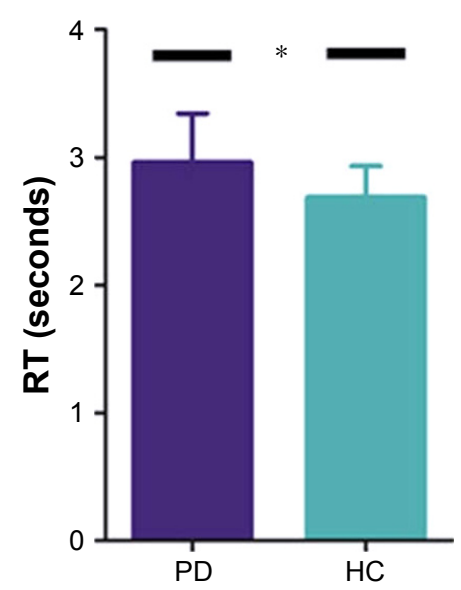

\section{B}
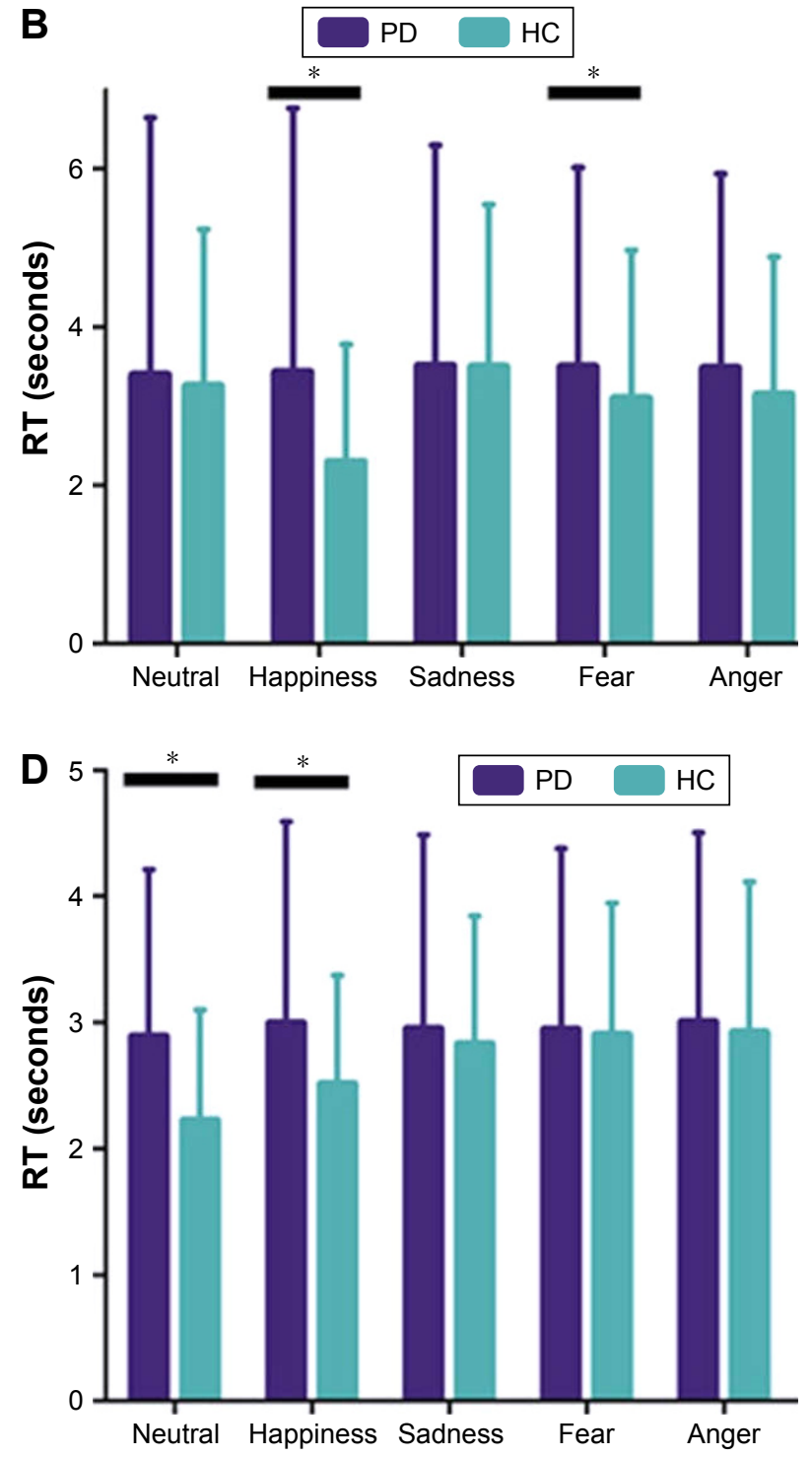

Figure 3 RT in the emotional expression test for the PD group (dark blue) and the HC group (light blue): (A) all emotions and (B) single emotion. Vocal emotion expression test for the PD group (dark blue) and the HC group (light blue): (C) all emotions and (D) single emotion. Shorter RT represents better performance. Data presented as mean \pm SD. Asterisks indicate a statistical difference between groups $(P<0.05)$.

Abbreviations: RT, reaction time; PD, Parkinson's disease; $\mathrm{HC}$, healthy control. 
Table 4 RT in facial and vocal expression

\begin{tabular}{|c|c|c|c|}
\hline RT (seconds) & $P D(n=15)$ & $H C(n=15)$ & $P$-values \\
\hline Facial total RT & $3.46 \pm 0.76$ & $3.05 \pm 0.45$ & 0.084 \\
\hline Neutral $(n=15)$ & $3.39 \pm 3.25$ & $3.25 \pm 1.98$ & 0.202 \\
\hline Happiness $(n=15)$ & $3.43 \pm 3.34$ & $2.29 \pm 1.48$ & $0.000 *$ \\
\hline Sadness $(n=15)$ & $3.5 \mathrm{I} \pm 2.79$ & $3.48 \pm 2.05$ & 0.054 \\
\hline Fear $(n=15)$ & $3.50 \pm 2.52$ & $3.10 \pm 1.87$ & $0.049 *$ \\
\hline Anger $(n=15)$ & $3.48 \pm 2.45$ & $3.14 \pm 1.74$ & 0.205 \\
\hline Vocal total RT & $2.95 \pm 0.39$ & $2.68 \pm 0.25$ & $0.004^{*}$ \\
\hline Neutral $(n=15)$ & $2.89 \pm 1.32$ & $2.24 \pm 0.87$ & $0.000 *$ \\
\hline Happiness $(n=15)$ & $3.00 \pm 1.60$ & $2.5 I \pm 0.86$ & $0.010^{*}$ \\
\hline Sadness $(n=15)$ & $2.95 \pm 1.54$ & $2.83 \pm 1.01$ & 0.240 \\
\hline Fear $(n=15)$ & $2.94 \pm 1.43$ & $2.90 \pm 1.05$ & 0.357 \\
\hline Anger $(n=15)$ & $3.00 \pm 1.50$ & $2.92 \pm 1.19$ & 0.879 \\
\hline
\end{tabular}

Notes: Data presented as mean \pm SD. Asterisks indicate a statistical difference between groups $(P<0.05)$.

Abbreviations: PD, Parkinson's disease; HC, healthy control; RT, reaction time. and reduced amplitude of facial muscles has been recorded during facial expressions. ${ }^{14}$ Throat muscles have also been found to be impaired in PD patients.

However, this impairment was not emotion specific, as each subject differed from the others. Inconsistent with previous research, PD patients exhibited a more extensive decline in emotional recognition and expression tasks, including negative (anger and sadness) and neutral emotions. ${ }^{8,9,26}$ The conflicting results were likely due to different methodologies and confounding factors (cognitive impairment and depressive state). When we controlled for these confounding factors, the results were consistent. ${ }^{10}$ In our study, there were no significant differences in neuropsychological characteristics
A

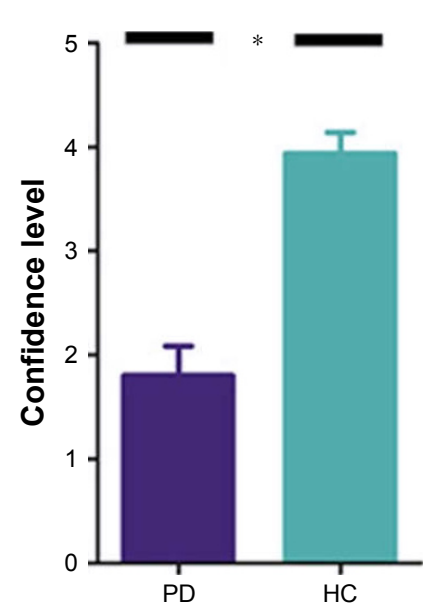

C

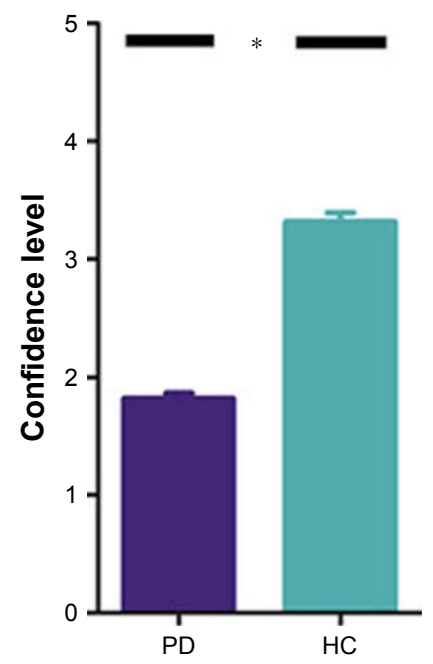

B

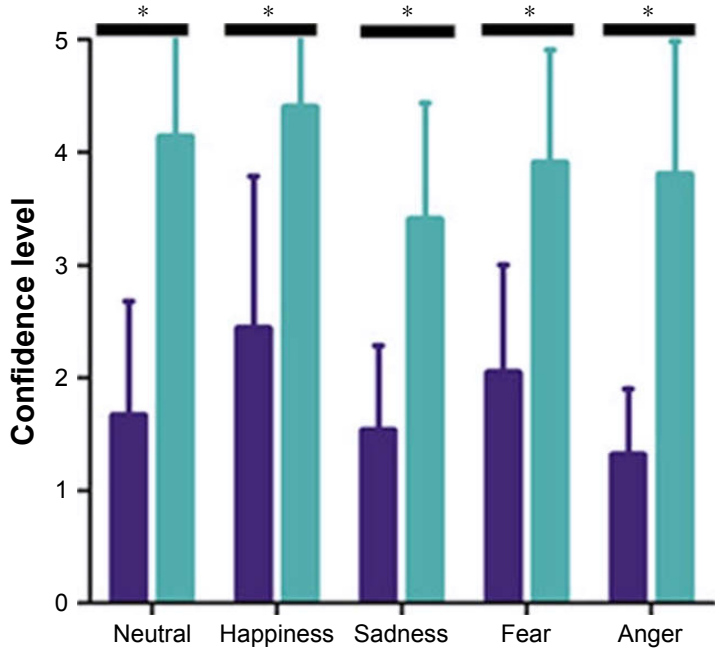

D

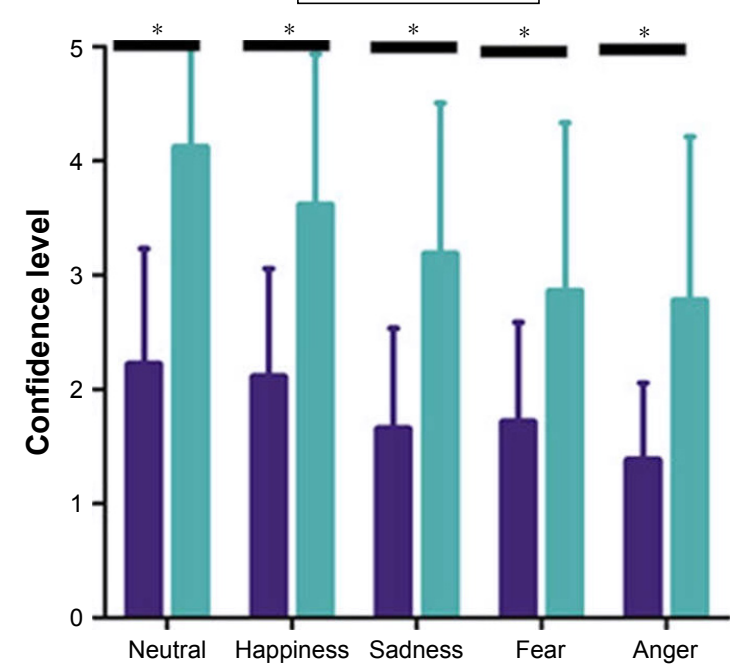

Figure 4 Confidence level in the emotional expression test for the PD group (dark blue) and the HC group (light blue): (A) all emotions and (B) single emotion. Vocal emotion expression test for the PD group (dark blue) and the HC group (light blue): (C) all emotions and (D) single emotion. Data presented as mean \pm SD. Asterisks indicate a statistical difference between groups $(P<0.05)$.

Abbreviations: PD, Parkinson's disease; HC, healthy control. 
Table $5 \mathrm{CL}$ in facial and vocal expression

\begin{tabular}{llll}
\hline $\mathbf{C L}$ & PD $(\mathbf{n}=\mathbf{I 5})$ & HC $(\mathbf{n}=\mathbf{I 5})$ & $P$-values \\
\hline Facial total $\mathrm{CL}$ & $1.80 \pm 0.29$ & $3.93 \pm 0.21$ & $0.000^{*}$ \\
Neutral $(\mathrm{n}=15)$ & $1.67 \pm 1.01$ & $4.14 \pm 1.06$ & $0.000^{*}$ \\
Happiness $(\mathrm{n}=15)$ & $2.44 \pm 1.35$ & $4.40 \pm 1.04$ & $0.000^{*}$ \\
Sadness $(\mathrm{n}=15)$ & $1.53 \pm 0.76$ & $3.40 \pm 1.04$ & $0.000^{*}$ \\
Fear $(\mathrm{n}=15)$ & $2.04 \pm 0.96$ & $3.91 \pm 1.00$ & $0.000^{*}$ \\
Anger $(\mathrm{n}=15)$ & $1.31 \pm 0.59$ & $3.80 \pm 1.18$ & $0.000^{*}$ \\
Vocal total $\mathrm{CL}$ & $1.81 \pm 0.20$ & $3.31 \pm 0.33$ & $0.000^{*}$ \\
Neutral $(\mathrm{n}=15)$ & $2.22 \pm 1.02$ & $4.15 \pm 1.25$ & $0.000^{*}$ \\
Happiness $(\mathrm{n}=15)$ & $2.11 \pm 0.95$ & $3.61 \pm 1.32$ & $0.000^{*}$ \\
Sadness $(\mathrm{n}=15)$ & $1.65 \pm 0.88$ & $3.18 \pm 1.33$ & $0.000^{*}$ \\
Fear $(\mathrm{n}=15)$ & $1.71 \pm 0.88$ & $2.85 \pm 1.48$ & $0.000^{*}$ \\
Anger $(\mathrm{n}=15)$ & $1.38 \pm 0.68$ & $2.77 \pm 1.44$ & $0.000^{*}$ \\
\hline
\end{tabular}

Notes: Data presented as mean \pm SD. Asterisks indicate a statistical difference between groups $(P<0.05)$.

Abbreviations: $\mathrm{PD}$, Parkinson's disease; $\mathrm{HC}$, healthy control; $\mathrm{CL}$, confidence level. between the two groups. Therefore, higher deficits in emotional processing in PD should be expected to be associated with increased pathological changes in additional anatomical structures as PD progresses. ${ }^{27-29}$

In addition, the participants' RT was correlated with their level of confidence in the emotional expression task. As expected, a shorter RT in evaluating the expression tasks resulted in a higher CL, in both the PD and HC groups.

Pearson's correlation test revealed a relationship between the ability to recognize emotions and the ability to express emotions in both facial and vocal emotion tasks. In a recent kinematic study, Bologna et al ${ }^{11}$ did not find any correlation between emotional recognition deficits and altered facial emotion expression kinematics in patients, suggesting that
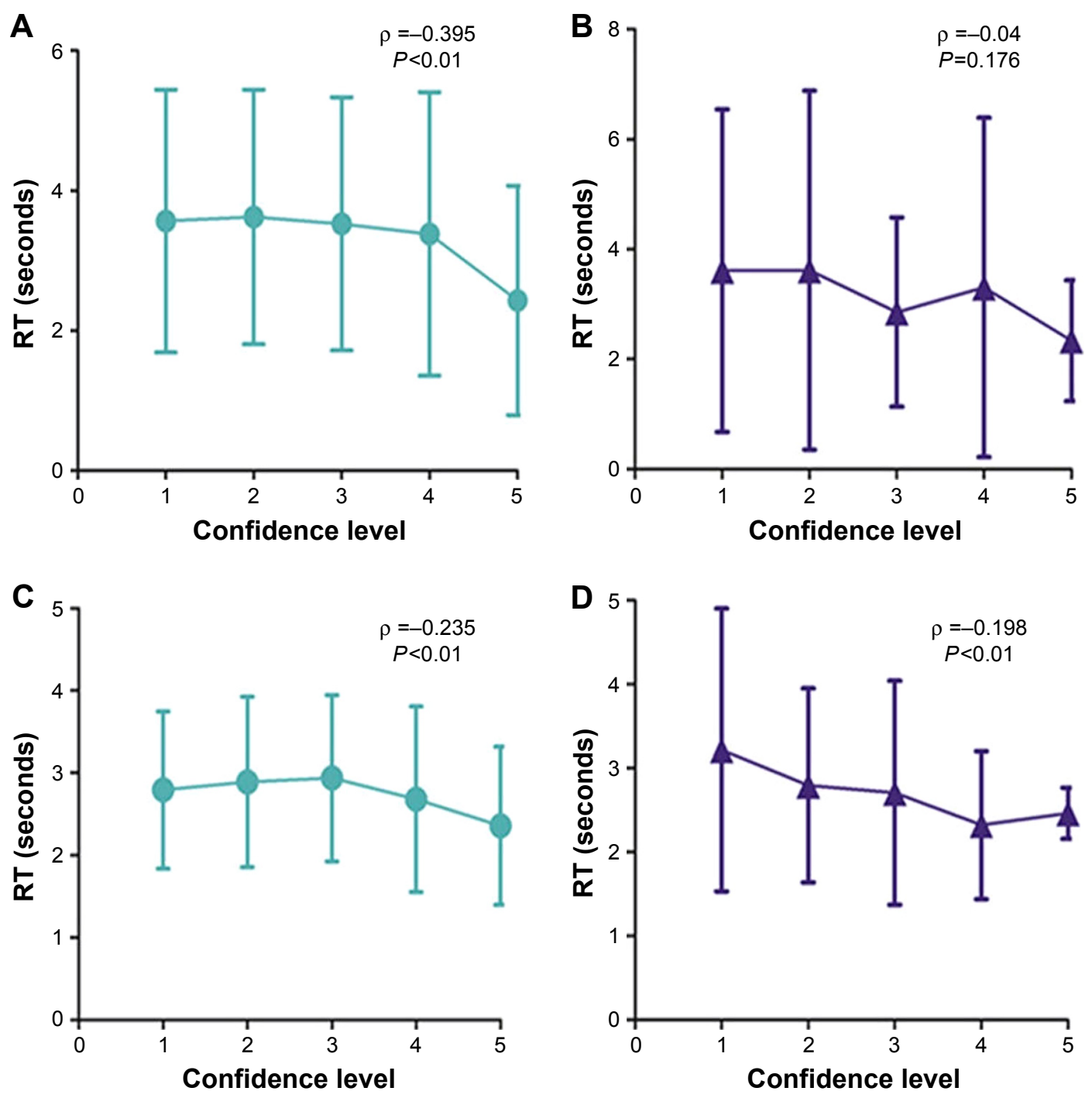

\section{$\mathrm{HC} \bigwedge \mathrm{AD}$}

Figure 5 Correlation between RT and confidence level: facial emotion expression test for (A) the HC group (light blue) and (B) the PD group (dark blue); vocal emotion expression test for (C) the HC group (light blue) and (D) the PD group (dark blue).

Abbreviations: RT, reaction time; HC, healthy control; PD, Parkinson's disease. 
Table 6 Correlation between RT and CL

\begin{tabular}{lllll}
\hline & Groups & Number & Spearman $\rho$ & $\boldsymbol{P}$-values \\
\hline Facial & HC & II 25 & -0.395 & $0.000^{*}$ \\
expression & PD & II 25 & -0.040 & 0.176 \\
Vocal & HC & II 25 & -0.235 & $0.000^{*}$ \\
expression & PD & II 25 & -0.198 & $0.000^{*}$ \\
\hline
\end{tabular}

Note: Asterisks indicate a statistical difference between groups $(P<0.05)$. Abbreviations: $\mathrm{RT}$, reaction time; $\mathrm{CL}$, confidence level; $\mathrm{HC}$, healthy control; $\mathrm{PD}$, Parkinson's disease.

these abnormalities are mediated by two separate pathophysiological mechanisms. The emotional recognition test used in that study was similar to the one used in this study, although the facial emotional expression methods were different. We believed that different methodologies led to the inconsistent results, since facial expressions of emotion were recorded using a three-dimensional optoelectronic system and analyzed using a facial action coding system in that study, while
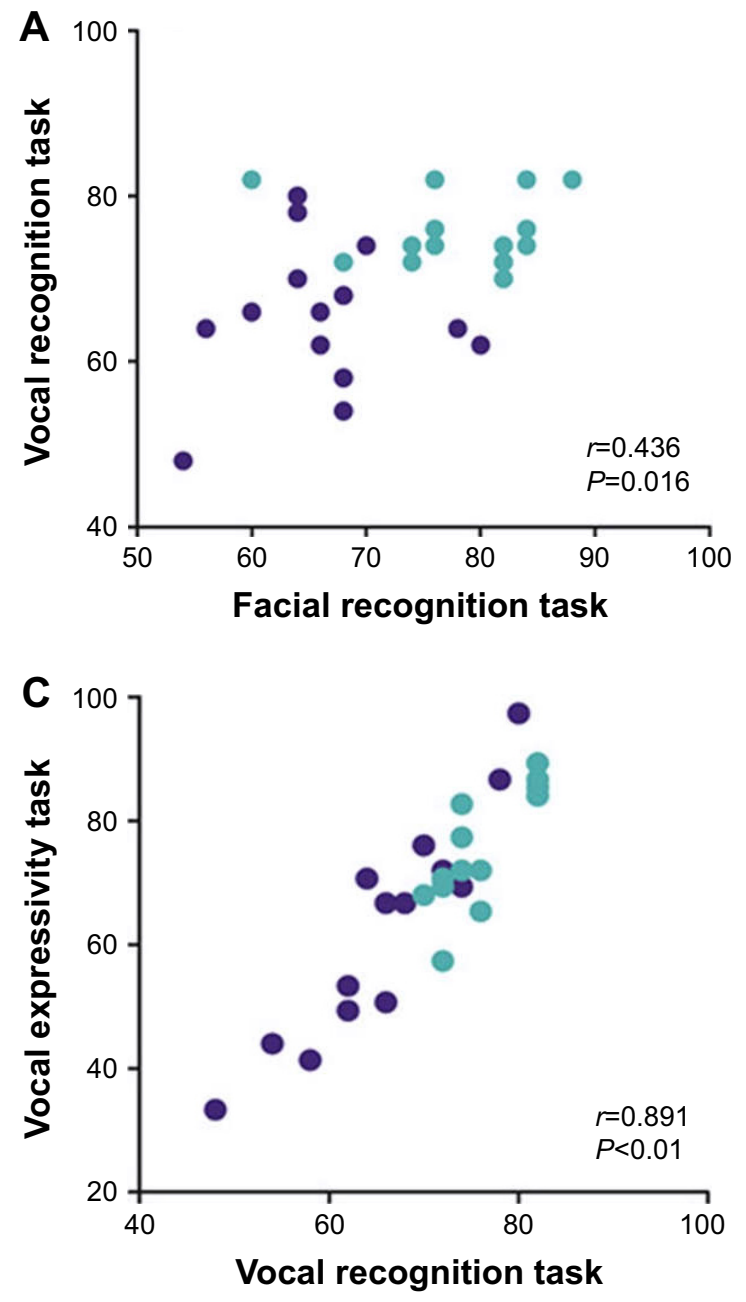

emotional expressions were analyzed by a group of healthy people according to their own understanding in this study. Several markers were placed on different parts of the face to track the kinematic features of the facial expressions (ie, the amplitude and peak velocity). However, multiple facial muscles are used while expressing emotions. This may have contributed to the different results. Previous studies have suggested the existence of "shared models", in which individuals mainly rely on processes that simulate the same emotional state in themselves when recognizing emotions expressed by others. ${ }^{10}$ These models are similar to mirror neurons, which have been demonstrated in the inferior frontal gyrus and can be activated by both observing and executing the same action. ${ }^{30,31}$ In addition, in a functional MRI study, while subjects were either observing or imitating facial emotional expressions, a largely similar network of brain areas was activated. ${ }^{32}$ Vocal emotional studies have also reached similar conclusions. ${ }^{33,34}$
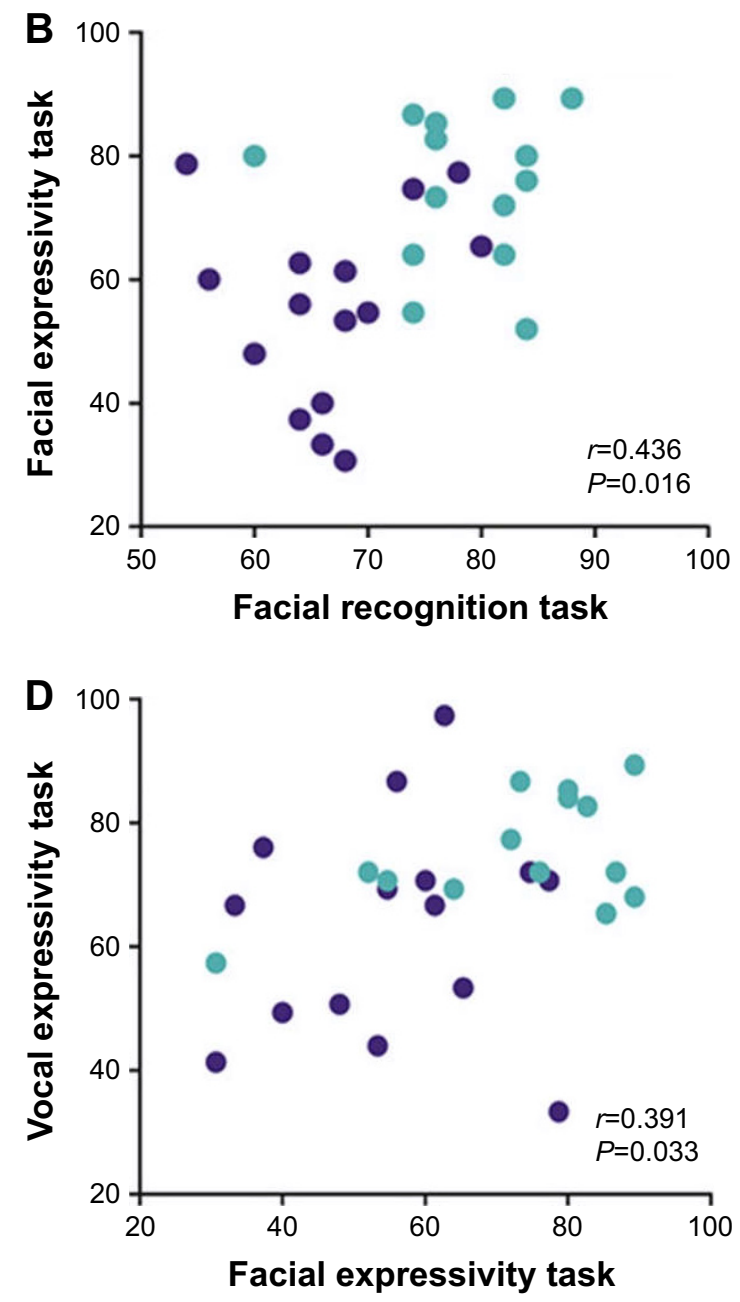

\section{$\mathrm{PD} \bigcirc \mathrm{HC}$}

Figure 6 Correlation between emotional recognition and emotional expression for the HC group (light blue) and the PD group (dark blue): (A) facial recognition task and vocal recognition task, (B) facial recognition task and facial expressivity task, (C) vocal recognition task and vocal expressivity task and (D) facial expressivity task and vocal expressivity task.

Abbreviations: HC, healthy control; PD, Parkinson's disease. 
Table 7 Correlation between emotional recognition and emotional expression

\begin{tabular}{llll}
\hline & Number & Pearson $\boldsymbol{r}$ & P-values \\
\hline $\begin{array}{l}\text { Facial recognition and } \\
\text { vocal recognition }\end{array}$ & 30 & 0.436 & $0.016^{*}$ \\
$\begin{array}{l}\text { Facial recognition and } \\
\text { facial expression }\end{array}$ & 30 & 0.436 & $0.016^{*}$ \\
$\begin{array}{l}\text { Vocal recognition and } \\
\text { vocal expression }\end{array}$ & 30 & 0.891 & $0.000^{*}$ \\
$\begin{array}{l}\text { Facial expression and } \\
\text { vocal expression }\end{array}$ & 30 & 0.391 & $0.033^{*}$ \\
\hline
\end{tabular}

Note: Asterisks indicate a statistical difference between groups $(P<0.05)$.

In this study, there was also relationship between facial and vocal tasks during both recognition and expression of emotions. This correlation is novel, but recent findings have suggested that facial and vocal emotional information interact with each other. ${ }^{35}$

However, there are certain limitations to this study. First, we recruited a relatively small sample. The influence of other factors, such as age, gender, or anti-PD drugs, was not specifically investigated. Studies have found a relation between levodopa and vocal performance: short-term levodopa administration or long-term dopaminergic medication administration can lead to a degree of improvement in vowel articulation in early PD. ${ }^{36,37}$ Second, PD is a clinically progressive disease and may have different subtypes. Thus, other stages (eg, the earlier or more advanced disease stages) or different disorder subtypes may have different impacts on emotional processing. Future studies should recruit patients with various PD stages and subtypes to clarify this issue. Third, we only evaluated posed expressions rather than spontaneous facial or vocal expressions. The pathophysiology of spontaneous emotional expressions may differ from that of posed expressions. Fourth, we did not conduct functional imaging, which prevents us from analyzing correlations between behavioral results and brain activation area. Finally, all the patients in our study were pre-DBS patients, and postoperative patients were excluded. Hence, we cannot compare emotional cognitive ability changes before and after surgery.

\section{Conclusion}

Our data provide novel information on altered emotional processing in pre-DBS PD using two sensory modalities. Facial emotion deficits and vocal emotion abnormalities were associated. The PD group exhibited poorer performance than the $\mathrm{HC}$ group on both tasks. In addition, our data allow us to speculate that emotional recognition and expression may share a common system.

\section{Abbreviations}

CL, confidence level; DBS, deep brain stimulation; HC, healthy control; KDEF, Karolinska Directed Emotional Faces; MAV, Montreal Affective Voices; PD, Parkinson's disease; RT, reaction time; STN, subthalamic nucleus.

\section{Acknowledgment}

$\mathrm{YJ}$ is grateful to Dr Guang Xie for the support in designing the study methods.

\section{Disclosure}

The authors report no conflicts of interest in this work.

\section{References}

1. Gee M, Dukart J, Draganski B, Wayne Martin WR, Emery D, Camicioli R. Regional volumetric change in Parkinson's disease with cognitive decline. J Neurol Sci. 2017;373:88-94.

2. Alexander GE, Crutcher MD. Functional architecture of basal ganglia circuits: neural substrates of parallel processing. Trends Neurosci. 1990; 13(7):266-271.

3. Alexander GE, DeLong MR, Strick PL. Parallel organization of functionally segregated circuits linking basal ganglia and cortex. Annu Rev Neurosci. 1986;9:357-381.

4. Middleton FA, Strick PL. Basal-ganglia "projections" to the prefrontal cortex of the primate. Cereb Cortex. 2002;12(9):926-935.

5. Le Jeune F, Péron J, Biseul I, et al. Subthalamic nucleus stimulation affects orbitofrontal cortex in facial emotion recognition: a PET study. Brain. 2008;131(Pt 6):1599-1608.

6. Chagas MHN, Tumas V, Pena-Pereira MA, et al. Neuroimaging of major depression in Parkinson's disease: cortical thickness, cortical and subcortical volume, and spectroscopy findings. J Psychiatr Res. 2017; 90:40-45.

7. Ruffman T, Henry JD, Livingstone V, Phillips LH. A meta-analytic review of emotion recognition and aging: implications for neuropsychological models of aging. Neurosci Biobehav Rev. 2008;32(4): 863-881.

8. Gray HM, Tickle-Degnen L. A meta-analysis of performance on emotion recognition tasks in Parkinson's disease. Neuropsychology. 2010; 24(2):176-191.

9. Péron J, Dondaine T, Le Jeune F, Grandjean D, Vérin M. Emotional processing in Parkinson's disease: a systematic review. Mov Disord. 2012; 27(2):186-199.

10. Ricciardi L, Visco-Comandini F, Erro R, et al. Facial emotion recognition and expression in Parkinson's disease: an emotional mirror mechanism. PLoS One. 2017;12(1):e0169110.

11. Bologna M, Berardelli I, Paparella G, et al. Altered kinematics of facial emotion expression and emotion recognition deficits are unrelated in Parkinson's disease. Front Neurol. 2016;7:230.

12. Albuquerque L, Martins M, Coelho M, et al. Advanced Parkinson disease patients have impairment in prosody processing. J Clin Exp Neuropsychol. 2016;38(2):208-216.

13. Rusz J, Cmejla R, Ruzickova H, Ruzicka E. Quantitative acoustic measurements for characterization of speech and voice disorders in early untreated Parkinson's disease. J Acoust Soc Am. 2011;129(1): 350-367.

14. Livingstone SR, Vezer E, McGarry LM, Lang AE, Russo FA. Deficits in the mimicry of facial expressions in Parkinson's disease. Front Psychol. 2016;7:780.

15. Graff-Radford J, Jones DT, Graff-Radford NR. Pathophysiology of language, speech and emotions in neurodegenerative disease. Parkinsonism Relat Disord. 2014;20 (Suppl 1):S49-S53. 
16. Wang JW, Zhang YQ, Zhang XH, Wang YP, Li JP, Li YJ. Cognitive and psychiatric effects of STN versus GPi deep brain stimulation in Parkinson's disease: a meta-analysis of randomized controlled trials. PLoS One. 2016;11(6): 0156721.

17. Brück C, Wildgruber D, Kreifelts B, Krüger R, Wächter T. Effects of subthalamic nucleus stimulation on emotional prosody comprehension in Parkinson's disease. PLoS One. 2011;6(4):e19140.

18. Williams NR, Foote KD, Okun MS. STN vs. GPi deep brain stimulation: translating the rematch into clinical practice. Mov Disord Clin Pract. 2014;1(1):24-35.

19. Keshtiari N, Kuhlmann M, Eslami M, Klann-Delius G. Recognizing emotional speech in Persian: a validated database of Persian emotional speech (Persian ESD). Behav Res Methods. 2015;47(1):275-294.

20. Calvo MG, Lundqvist D. Facial expressions of emotion (KDEF): identification under different display-duration conditions. Behav Res Methods. 2008;40(1):109-115.

21. Kalampokini S, Lyros E, Luley M, et al. Facial emotion recognition in Parkinson's disease: association with age and olfaction. J Clin Exp Neuropsychol. 2017:1-11.

22. Belin P, Fillion-Bilodeau S, Gosselin F. The Montreal Affective Voices: a validated set of nonverbal affect bursts for research on auditory affective processing. Behav Res Methods. 2008;40(2):531-539.

23. Reznikov R, Bambico F, Diwan M, et al. Prefrontal cortex deep brain stimulation improves fear and anxiety-like behaviour and reduces basolateral amygdala activity in a preclinical model of post-traumatic stress disorder. Neuropsychopharmacology. Epub 2017 Sep 1.

24. Maren S. Synapse-specific encoding of fear memory in the amygdala. Neuron. 2017;95(5):988-990.

25. Schultz DH, Balderston NL, Baskin-Sommers AR, Larson CL, Helmstetter FJ. Corrigendum: psychopaths show enhanced amygdala activation during fear conditioning. Front Psychol. 2017;8:1457.

26. Assogna F, Pontieri FE, Caltagirone C, Spalletta G. The recognition of facial emotion expressions in Parkinson's disease. Eur Neuropsychopharmacol. 2008;18(11):835-848.
27. Braak H, Del Tredici K. Invited article: nervous system pathology in sporadic Parkinson disease. Neurology. 2008;70(20):1916-1925.

28. Halliday GM, McCann H. The progression of pathology in Parkinson's disease. Ann N Y Acad Sci. 2010;1184:188-195.

29. Halliday G, Hely M, Reid W, Morris J. The progression of pathology in longitudinally followed patients with Parkinson's disease. Acta Neuropathol. 2008;115(4):409-415.

30. Rizzolatti G, Arbib MA. Language within our grasp. Trends Neurosci. 1998;21(5):188-194.

31. Rizzolatti G, Fadiga L, Gallese V, Fogassi L. Premotor cortex and the recognition of motor actions. Brain Res Cogn Brain Res. 1996;3(2): 131-141.

32. Carr L, Iacoboni M, Dubeau MC, Mazziotta JC, Lenzi GL. Neural mechanisms of empathy in humans: a relay from neural systems for imitation to limbic areas. Proc Natl Acad Sci US A. 2003;100(9):5497-5502.

33. Liebenthal E, Silbersweig DA, Stern E. The language, tone and prosody of emotions: neural substrates and dynamics of spoken-word emotion perception. Front Neurosci. 2016;10:506

34. Huang X, Chen X, Yan N, et al. The impact of Parkinson's disease on the cortical mechanisms that support auditory-motor integration for voice control. Hum Brain Mapp. 2016;37(12):4248-4261.

35. Ho HT, Schröger E, Kotz SA. Selective attention modulates early human evoked potentials during emotional face-voice processing. $J \operatorname{Cog} n$ Neurosci. 2015;27(4):798-818.

36. Skodda S, Visser W, SchlegelU. Short-and long-term dopaminergic effects on dysarthria in early Parkinson's disease. J Neural Transm (Vienna). 2010;117(2):197-205.

37. Rusz J, Tykalová T, Klempír J, Čmejla R, Růžička E. Effects of dopaminergic replacement therapy on motor speech disorders in Parkinson's disease: longitudinal follow-up study on previously untreated patients. J Neural Transm (Vienna). 2016;123(4):379-387.
Neuropsychiatric Disease and Treatment

\section{Publish your work in this journal}

Neuropsychiatric Disease and Treatment is an international, peerreviewed journal of clinical therapeutics and pharmacology focusing on concise rapid reporting of clinical or pre-clinical studies on a range of neuropsychiatric and neurological disorders. This journal is indexed on PubMed Central, the 'PsycINFO' database and CAS,

\section{Dovepress}

and is the official journal of The International Neuropsychiatric Association (INA). The manuscript management system is completely online and includes a very quick and fair peer-review system, which is all easy to use. Visit http://www.dovepress.com/testimonials.php to read real quotes from published authors. 